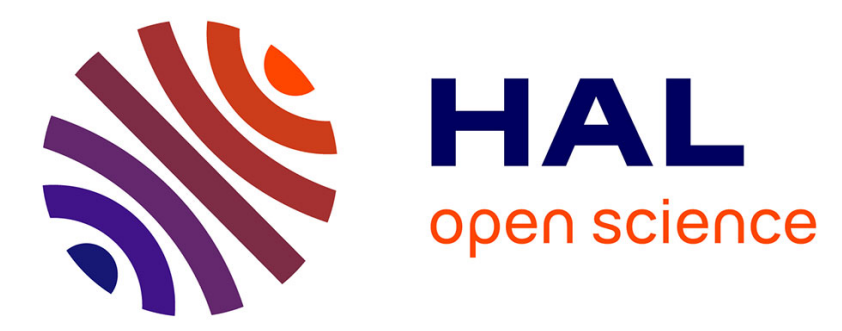

\title{
L'art et l'Etat au début de la IIIe République, ou les conditions d'impossibilité de la mise en forme d'une politique.
}

Vincent Dubois

\section{- To cite this version:}

Vincent Dubois. L'art et l'Etat au début de la IIIe République, ou les conditions d'impossibilité de la mise en forme d'une politique.. Genèses. Sciences sociales et histoire, 1996, 23, p. 6-29. halshs00488921

\section{HAL Id: halshs-00488921 https://shs.hal.science/halshs-00488921}

Submitted on 3 Jun 2010

HAL is a multi-disciplinary open access archive for the deposit and dissemination of scientific research documents, whether they are published or not. The documents may come from teaching and research institutions in France or abroad, or from public or private research centers.
L'archive ouverte pluridisciplinaire HAL, est destinée au dépôt et à la diffusion de documents scientifiques de niveau recherche, publiés ou non, émanant des établissements d'enseignement et de recherche français ou étrangers, des laboratoires publics ou privés. 


\section{L'art et l'Etat au début de la IIIe République, ou les conditions d'impossibilité de la mise en forme d'une politique ${ }^{i}$}

Vincent Dubois

Genèses, sciences sociales et histoire, 23, Juin 1996, p. 6-29

Un préjugé négatif pèse sur l'intervention de l'Etat dans le domaine artistique sous la IIIe République. Les pouvoirs publics manqueraient à leurs «devoirs» en la matière, en n'aidant pas assez les artistes, en ne réalisant pas les institutions et les investissements nécessaires au développement artistique du pays, en maintenant une administration inefficace aux mains de fonctionnaires et sous l'autorité de responsables politiques tous aussi incultes que vaniteux. Quelques «affaires», comme le refus du legs Caillebote en 1894, le vol de la Joconde au Louvre en 1911, ou la «médiocrité» rituellement dénoncée des œuvres résultant de commandes d'Etat fourniraient autant de preuves à l'appui d'un jugement maintes fois proféré : l'Etat n'a «pas de politique» pour les Beaux-Arts. Pire : les dirigeants de la République seraient responsables de la «corruption du goût français». La dénonciation est formulée par nombre de commentateurs de l'époque. Elle est réactualisée périodiquement par les agents qui militent en faveur d'une «vraie» politique, la période de la IIIe République étant alors constituée en contre-exemple absolui ${ }^{\text {ii }}$ Elle est enfin reproduite par nombre d'auteurs qui cherchent à rendre compte, sur le long terme, de l'histoire de l'intervention «culturelle» de l'Etat ${ }^{\mathrm{iii}}$.

Plusieurs historiens ont invité à réviser un tel jugement. Cherchant à contrebalancer les réquisitoires systématiques et parfois sommaires, ils ont déployé les arguments de la défense. Le label stigmatisant d'«art officiel» a été remis en cause, à la faveur du regain d'intérêt esthétique pour les œuvres ainsi désignées. Dans le prolongement de cette redécouverte, l'action des agents du champ politico-administratif pour les Beaux-Arts a pu 
bénéficier d'une bienveillance inédite ${ }^{\text {iv }}$. L'exaltation des «sources» de l'idéal républicain a conduit à réévaluer l'investissement artistique public des années 1870-1940, mettant en avant sa cohérence et sa «modernité» ${ }^{\mathrm{V}}$. La référence à la IIIe République a même été mobilisée à l'inverse de ce qu'elle était par les militants de l'action culturelle : non plus pour faire valoir la nécessité d'une rupture, mais pour affirmer celle d'un retour ${ }^{\mathrm{vi}}$.

Dénonciation ou réhabilitation : dans un cas comme dans l'autre, l'évocation des rapports entre l'art et la politique sous la IIIe République témoigne d'un rapport ambigu à l'histoire. Dénoncer l'absence de politique ou au contraire en exhumer ex post les origines lointaines, c'est dans les deux cas présupposer l'existence transhistorique d'une «mission» des pouvoirs publics à l'égard des choses de l'art. C'est autrement dit oublier le caractère historiquement construit des «fonctions de l'Etat», et de catégories - comme celle de «politique culturelle» - qui ont été progressivement naturalisées. Stigmatiser l'incurie des responsables politiques et de l'administration comme à l'inverse faire valoir la diligence avec laquelle ils s'acquittent de leur tâche, c'est par ailleurs supposer que l'intervention publique repose sur la «volonté» d'hommes censés la conduire. C'est autrement dit oublier ce que ces «volontés» ou leur absence doivent à la configuration des rapports de force qui les rendent possibles ou non.

Restituer l'historicité d'une intervention publique qui paraît aujourd'hui «naturelle»; retracer les luttes des champs politique et artistique dans lesquelles se joue la possibilité d'une «politique artistique»: c'est à ce double programme qu'on souhaite apporter une contribution. On voudrait dans cette perspective montrer que les inclinations opposées à la dénonciation et à la réhabilitation trouvent leurs origines dans cela même dont il faut rendre compte, et dont elles organisent l'oubli : les conditions d'impossibilité de la mise en forme d'une «politique» dans le domaine des Beaux-Arts dans la conjoncture des années 18901910.

Faut-il le rappeler, le stade auquel parvient la genèse de l'Etat en France à cette période est bien loin de permettre l'imposition de l'intervention publique dans des domaines multiples et variés comme une évidence. Cette intervention est d'autant moins évidente dans les espaces sociaux qui, comme le champ artistique, affirment alors leur autonomie : elle peut 
dans ce cas être efficacement dénoncée comme une ingérence illégitime. Plus encore, le travail d'autonomisation du champ artistique conduit à imposer la représentation de l'art et de l'Etat comme deux choses par nature contraires l'une à l'autre. Cette représentation socialement construite produit des effets qui contribuent à la réaliser. En structurant les visions du monde social, elle détermine de fait l'espace des actions possibles, y compris celles des agents qui représentent et font exister l'Etat. Ces agents, placés en situation de porte-à-faux, réalisent par la faiblesse de leur investissement et les incertitudes de leurs pratiques la prophétie de leur illégitimité à intervenir. Illégitimité intériorisée par les agents, elle est aussi objectivée dans des positions et des institutions précaires. Plus qu'une «politique», c'est alors l'illégitimité de cette «politique» qui est institutionnalisée. Les fluctuations et l'instabilité des constructions et positions institutionnelles confèrent en effet l'objectivité des choses et des hommes à la représentation historiquement construite d'un Etat «incompétent» ${ }^{\text {vii }}$.

\section{L'art et l'Etat : la construction d'un antagonisme}

Tout comme l'art et l'argent ${ }^{\mathrm{viii}}$, l'art et l'Etat ont été opposés comme des contraires s'excluant mutuellement. Cette opposition irréductible est liée au travail d'autonomisation du champ artistique. Le fait même de prétendre à l'appartenance à ce champ implique en effet de dénoncer toute intrusion susceptible d'en menacer l'indépendance. Ces dénonciations débouchent sur une forme de censure inversée : les paroles et les actes des agents du champ politique et de l'Etat sont en quelque sorte frappés d'indignité.

\section{Séparer les Beaux-Arts de l'Etat}

Dans la conjoncture particulière du tournant du siècle, le caractère récent de l'autonomie du champ artistique conduit à exacerber la résistance à l'égard de tout ce qui peut apparaître comme l'imposition de principes hétéronomes. Les clivages internes sont -provisoirement et partiellement- oubliés dès lors qu'il s'agit de garantir le respect des frontières 
communes. Nonobstant les différences objectives qui séparent les agents du champ artistique, les prises de position publiques des artistes à l'égard de l'intervention publique se rejoignent dans une même hostilité.

Le cas de la peinture, au centre des débats sur l'art et l'Etat, permet de le montrer. Les rapports qu'entretiennent les différentes catégories d'artistes avec les institutions publiques sont nettement différenciés : de la concurrence à la collusion pour les artistes académiques ; de l'ignorance à l'opposition ouverte pour les artistes dits libres, des relations proches de celles qui lient des fournisseurs à leurs clients pour les artistes dits officiels ${ }^{\mathrm{ix}}$. Néanmoins, malgré leurs différences et les écarts de position qui séparent les artistes qui y appartiennent, aucun de ces trois groupes n'est le lieu d'un appel à l'intervention publique ni de la production de discours ou de pratiques susceptibles de la légitimer. A l'inverse, chacun d'eux suscite des prises de position de défiance à cet égard.

On le comprend aisément pour ce qui concerne les artistes «libres»: regroupant des tendances aussi éloignées que l'art social ou l'art pour l'art, cette catégorie trouve son unité dans l'opposition commune aux institutions, académiques mais aussi étatiques. Par défense farouche de l'autonomie du champ artistique et/ou par nécessité faite vertu, ils refusent tout soutien public et dénoncent à l'envi la médiocrité des achats et des productions aidées par l'Etat.

La position des artistes académiques est plus complexe. S'ils sont présents dans les instances qui organisent l'intervention publique - comme le Conseil supérieur des beauxarts créé en 1875 - ils ne considèrent pas qu'elle soit a priori légitime, et moins encore que les agents de l'Etat soient autorisés à la définir. Ils cherchent, pour perpétuer le respect des canons académiques, à les faire prévaloir dans les décisions et au sein des établissements publics (enseignement des Ecoles des Beaux-Arts, collections des musées, décoration des édifices publics...) et sont prompts à dénoncer tout écart par rapport à ces normes. Plus que la légitimité de l'intervention publique, c'est sa surveillance qu'ils organisent. Revendiquant la position exclusive d'experts, ils dénient non seulement ces prérogatives aux artistes nonacadémiques, mais dénoncent l'ignorance et l'illégitimité des agents du champ politicoadministratif qui prétendraient s'occuper d'art. 
La position des artistes «officiels» envers le commanditaire public dont ils dépendent est plus ambiguë encore. Loin de se faire les défenseurs et les théoriciens de l'intervention des pouvoirs publics, ils critiquent volontiers les dangers de l'ingérence étatique. C'est que leur position particulière ne les place pas en dehors du champ artistique, et ne les fait donc pas échapper à ses règles, dont la défiance à l'égard de toute «intrusion» compte parmi les plus communément admises. Les artistes qu'on peut objectivement définir comme «officiels» ou «commerciaux» en raison de leur proximité aux règles des institutions publiques ou du champ économique, refusent de se laisser étiqueter comme tels : dès lors que sont imposés les principes de l'autonomie du champ artistique, ces désignations valent dénonciation. Tout comme l'intégration au marché privé n'exclut pas la dénégation de l'économie, participer aux procédures publiques n'empêche pas de prendre position contre toute intervention de l'Etat. Pour ne prendre qu'un exemple, le peintre Raffaëlli, qu'Albert Boime cite comme l'un des principaux représentants du groupe de «l'art officiel», n'hésite pas à réclamer, avec d'autres artistes et intellectuels aux positions parfois très éloignées, l'absolue nécessité de «séparer l'art et l'Etat ${ }^{\mathrm{x}} »$.

Depuis les différents pôles du champ artistique, les artistes convergent donc vers une même hostilité à l'Etat; plus encore, cette convergence prend forme dans des mobilisations collectives. En témoignent les résultats d'une «Enquête sur la séparation des Beaux-Arts et de l'Etat» publiée dans une petite revue en 1904, qui réunit les réponses d'une cinquantaine $\mathrm{d}$ 'artistes et intellectuels - auxquels s'ajoutent quelques rares parlementaires ${ }^{\mathrm{xi}}$.

S'inscrivant dans un débat plus vaste, périodiquement réactivé dans la presse et les revues intellectuelles lors des discussions budgétaires ou du Salon annuel notamment, cette enquête est explicitement destinée à établir l'illégitimité de l'intervention publique. Pour les artistes et intellectuels qui y répondent, c'est une façon d'intervenir dans le débat politique. Le commentaire qui suit la publication des différentes opinions le montre bien. De cette manière, «la question de la séparation des Beaux-Arts et de l'Etat va se poser devant nos législateurs. [...] C'est à leur usage que nous avons réuni cet ensemble d'opinions», proclame Maurice Le Blond. «Cet ensemble d'opinions», dont parle l'enquêteur, est mis en scène de manière à constituer à la fois un groupe mobilisé cherchant à faire valoir un point 
de vue collectif auprès des instances de représentation politique, et l'équivalent d'une commission parlementaire, experte et fortement investie, substituée à celles, «incompétentes» voire «désinvoltes» de la Chambre des députés. C'est, en quelque sort, un «parlement des artistes» qui est ainsi constituéxii .

«Nous croyons être l'interprète de tout le public artiste et lettré de France, en demandant que la prochaine discussion du budget des Beaux-Arts ne soit pas escamotée, comme à l'ordinaire, suivant la déplorable coutume qui existe au Palais-Bourbon. Nos politiciens, en effet, ne manquent jamais de traiter les questions d'Art avec une insouciance dédaigneuse, avec la plus extrême désinvolture» ${ }^{\text {xiii }}$.

Les partisans de la «séparation» sont nombreux et peuvent mobiliser de fermes principes pour affirmer leur hostilité à l'intervention publique. Outre les principes politiques, qui de l'anarchisme de Jean Grave au libéralisme de Quentin-Bauchart permettent de plaider pour la suppression du budget des Beaux-Arts en même temps que tous les budgets de l'Etat, ce sont surtout les principes du champ artistique qui fondent une opposition à l'intervention publique manifestée depuis les différents pôles du champ. C'est ainsi que le peintre Georges Desvallières, exaltant la mythologie de l'artiste maudit, affirme que «les grandes œuvres d'art ne sortiront jamais que d'une Ecole, celle de la Peine et souvent de la misère, car comme la guerre fait les héros, c'est la souffrance qui fait l'artiste», montrant par là l'impossibilité logique d'une aide publique à l'art ${ }^{\mathrm{xiv}}$. Plus, c'est l'affirmation même des principes -ou des mythes- fondateurs de l'autonomie du champ artistique qui conduit à produire la représentation d'un antagonisme irréductible entre l'art et l'Etat. Du dramaturge Gabriel Trarieux qui affirme que «de toutes les intrusions de l'Etat, celle qui lèse l'Art est la plus fâcheuse et la plus ridicule» au compositeur Vincent d'Indy qualifiant de «monstruosité» l'enseignement de l'art par l'Etat et à Willette, critique et ancien élève des Beaux-Arts qui résume : «l'Art c'est la liberté, l'Etat c'est la tyrannie», les artistes fondent leur position même sur l'imposition réussie de la représentation du rapprochement de l'art et de l'Etat comme une alliance contre-nature. De l'autre côté, la défense de cette intervention, provenant d'agents dominés du champ littéraire ou de parlementaires, est rare, prudente ou formulée sur un mode ironique qui la retourne en dénonciation.

L'enquêteur transpose les règles en vigueur dans le champ politique et fait de l'enquête l'équivalent d'un vote. Il peut alors affirmer qu'une «nette majorité» se dégage «contre 
l'imposition d'un Art d'Etat», et termine en se félicitant de la «condamnation unanime» des liens entre l'Art et l'Etat. Au vu de la présentation et des questions posées, de telles conclusions ne surprennent guère. En effet, c'est sur le double registre des principes et de la dénonciation de l'Etat que l'enquête est placée. Les débats du moment sur la séparation de l'Eglise et de l'Etat permettent une analogie propice à la manipulation de grandes idées, dans le sens d'une dissociation, à l'image du traitement de la question religieuse, des sphères artistique et étatique. La référence aux débats qui aboutissent, un an plus tard, à la loi de séparation, prend d'autant plus de poids que la métaphore religieuse est particulièrement prégnante lorsqu'il s'agit d'art. Si l'on admet le principe général de la neutralité religieuse de l'Etat, pourquoi le «culte de l'art» ferait-il exception ? Filer la métaphore permet alors de dénoncer le non respect de ce principe de neutralité : le monopole de l'Etat sur l'enseignement artistique, inévitablement soumis aux normes d'un académisme dévoyé, aboutit à la suprématie «d'une véritable Eglise d'art» qui dispense son «catéchisme esthétique». L'analogie donne du poids aux arguments ; elle permet également de généraliser le propos, et de situer l'enquête au niveau, cette fois, d'une discussion générale sur «le bon régime». «Haute question de principes» s'il en est, les rapports de l'art et de l'Etat permettent plus généralement de traiter dans son ensemble du système politique et social. Faut-il privilégier l'autorité ou la liberté ? Comment préserver la justice ? Quel est le rôle d'un Etat démocratique? Autant de questions qui permettent, par un glissement et un élargissement progressif, l'expression légitime d'artistes sur les questions politiques les plus nobles et les plus générales.

Mais la mise en question du rôle de l'Etat ne se cantonne pas à la sphère des grands systèmes philosophiques. L'Etat n'est pensé qu'en référence à un «système des Beaux-Arts», pour employer une expression plus tardive, fait de «vanité» et de pesanteur bureaucratique. Même lorsqu'il est qualifié d'«Etat-Mécène», il est montré comme une mécanique administrative aux antipodes de l'art: «un être abstrait et sans entrailles, une entité dépourvue de sensibilité et de culture, [...] un despote bureaucratique, anonyme et irresponsable qui n'est capable que de déterminer le triomphe de la Médiocrité et de la 
Laideur ${ }^{\mathrm{xv}}{ }^{\text {. Pire, }}$ c'est une machine exclusivement négative et coercitive, qui «monopolise» les moyens, «impose» sa conception esthétique et «réprime»les autres.

\section{La production de l'incompétence artistique des agents de l'Etat}

L'autonomisation du champ artistique et l'imposition réussie de la représentation de l'art et de l'Etat comme deux choses par nature étrangères l'une à l'autre conduisent à une censure inversée : les agents de l'Etat voient leurs actes soumis à l'examen sans concession de ceux qui prétendent avec succès au monopole de l'autorité artistique. Quelles que soient par ailleurs les différences objectives qui les séparent du point de vue de la compétence artistique, parlementaires, ministres ou fonctionnaires qui prétendent intervenir dans les affaires intérieures du champ artistique sont réunis par les artistes dans une commune illégitimité à traiter des choses de l'art. Il suffit pour s'en convaincre de restituer les critiques dont font l'objet les principales formes d'objectivation de l'intervention artistique publique: administration des Beaux-Arts, débats parlementaires, postes ministériels consacrés aux Beaux-Arts.

L'administration des Beaux-Arts se trouve au centre de l'antagonisme entre l'art et l'Etat construit par les artistes. Par sa dénomination même, elle porte au persiflage et à la dénonciation: la contradiction qui la fonde est pointée de manière récurrente dans les discours des artistes, la critique et la presse de manière plus générale. C'est ainsi que le chef de cette administration, le Directeur des Beaux-Arts, se voit régulièrement dénoncé pour l'absurdité de son titre : «"Serviteur” des Beaux-Arts ne sonne-t-il pas avec plus de décence et moins de démence ? On ne "dirige" pas les Beaux-Arts, on les "sert"..> L'intervention dans le domaine de l'art des parlementaires et responsables gouvernementaux n'est pas mieux considérée que celle de l'administration. Au-delà des origines sociales -bourgeois de province, représentants de départements ruraux, etc.- qui expliqueraient l'«ignorance» des hommes politiques, c'est par le fait même qu'ils prétendent parler d'art de l'extérieur du champ artistique que parlementaires et ministres s'attirent l'ironie et la défiance des commentateurs autorisés. Si la plupart des 
parlementaires se rendent coupables d'indifférence ou de manque d'enthousiasme, ceux qui tentent d'y échapper ne sont pas reconnus pour autant. Leurs éventuels investissements artistiques ne sont ainsi apparemment pas payés de retour. Rares sont les députés qui ont tiré une véritable reconnaissance de leurs interventions dans ce domaine. Il en est en revanche de nombreux - tel le député Cousset dans les années 1880-1890 - qui se sont fait connaître par l'inconsistance de leurs propos sur l'art.

Les responsables gouvernementaux chargés des Beaux-Arts subissent pour la plupart les mêmes attaques. Loin de marquer l'établissement d'un lien entre l'art et l'Etat, ces postes ministériels et leurs titulaires sont mobilisés comme une preuve souvent brandie et régulièrement moquée de l'impossibilité et/ou du ridicule de ce lien. L'accusation d'incompétence dont font l'objet les responsables ministériels chargés des Beaux-Arts tout au long de la Troisième République est de fait un leitmotiv répété par les commentateurs de l'époque. Des agents aux positions importantes dans le champ artistique, comme Frantz Jourdain (président du Syndicat de la presse artistique et du Salon d'automne), profèrent des prédictions définitives qui ne sont pas sans liens avec leur réalisation effective : «le suffrage universel placera toujours un ancien vaudevilliste ou un vague normalien au Ministère des Beaux-Arts» ${ }^{\text {xvii }}$.

Que les pratiques parlementaires ou ministérielles dans le domaine des Beaux-Arts soient stigmatisées par le fait même qu'elles émanent d'agents du champ politique est indiqué par la réduction systématique à des «tactiques politiciennes» qu'elles subissent de la part des agents socialement autorisés à parler d'art. Une «déplorable coutume qui existe au PalaisBourbon» fait que la discussion du budget des Beaux-Arts est le plus souvent «escamotée» ${ }^{\text {xviii }}$. Si ce n'est pas le cas, c'est pour servir d'autres fins que la «cause de l'art». Ainsi, les prises de position - nombreuses - hostiles au financement public de telle ou telle institution artistique ne sont là que pour flatter les «bas instincts» des électeurs «peu éclairés»; elles ne constituent rien de plus que la recherche d'une «satisfaction des appétits purement électoraux se cachant sous des dehors artistiques ${ }^{\mathrm{xix}} \gg$.

«L'assaut du sous-secrétariat d'Etat aux Beaux-Arts, au profit de toutes les médiocrités de leurs arrondissements, a été incessant de la part de presque tous les députés. Ceux qui se sont occupés des intérêts généraux de l'art, de la plus noble, de la plus glorieuse source de prospérité et d'influence du pays, sont toujours restés à l'état d'infime minorité ; sur ce terrain-là, il a toujours 
fallu forcer la main à la majorité ${ }^{\mathrm{xx}} \gg$ Lorsque ce n'est pas le cas, il ne faut pas pour autant en déduire un intérêt soudain pour les questions artistiques : simplement, les «politiciens» utilisent le chapitre des Beaux-Arts, comme n'importe quel autre, à des fins tactiques. Autant dire que la discussion n'est guère élevée; les pratiques du log-rolling et de l'obstruction ne peuvent qu'engendrer la vacuité des débats. «La discussion du budget dure parfois quelques instants, parfois des semaines entières. La Chambre veut ou ne veut pas en finir selon qu'elle est favorable au Gouvernement ou cherche à l'acculer à des douzièmes provisoires» ${ }^{\mathrm{xxi}}$.

Une réduction homologue à des tactiques politiciennes est opérée à propos du poste de secrétaire d'Etat aux Beaux-Arts et de ses titulaires. Ce poste ne serait rien d'autre qu'une ressource utile à la constitution d'une coalition gouvernementale : il devrait moins son existence au nécessaire accomplissement d'une «fonction» artistique de l'Etat qu'à la logique partisane de l'équilibrage et du partage des postes. Dès lors, son titulaire ne peut être que placé là par le hasard de combinaisons obscures, et donc indépendamment des compétences requises pour l'exercice de cette charge.

L'état du champ artistique - en phase d'affirmation de l'autonomie - et des rapports entre les champs artistique et politique - distanciation croissante et multiplication des interventions politiques des artistes - conduit à ce qu'au tournant du siècle, l'art et l'Etat soient construits, par les artistes, comme deux choses inconciliables. Cet antagonisme s'impose avec d'autant plus de force qu'il n'est pas seulement le produit d'une différenciation - telle que celle qu'opèrent les tenants de l'art pour l'art en refusant toute implication politique et sociale - mais aussi celui de la concurrence qui apparait entre les artistes et les agents de l'Etat pour le monopole de l'universel ${ }^{\mathrm{xxi}}$. L'on comprend dès lors que l'intervention de l'Etat dans le domaine artistique puisse être placée dans une situation de porte-à-faux, et ce d'autant plus que cette intervention peut par ailleurs être dénoncée comme contraire aux principes sur lesquels se construit l'Etat républicain ${ }^{\text {xxiii. }}$.

\section{L’intériorisation de l'illégitimité}

Ces représentations en elles-mêmes ne sauraient être tenues pour le principe actif de l'empêchement d'une «politique artistique»; elles ne revêtent une efficacité qu'en tant qu'elles sont intériorisées par les agents censés produire cette «politique». A l'état intériorisé, les représentations de l'art et de l'Etat comme couple d'antonymes et d'une 
intervention publique par essence illégitime peuvent, cette fois, produire des effets pratiques. Elles obligent les agents du champ politique à la prudence, et confèrent à leurs discours un statut incertain.

\section{L'art et l'Etat : un sujet «délicat»}

Les Beaux-Arts sont une matière délicate, au double sens du terme : si les considérations générales sur les arts et, en particulier, la question de leur rapport à l'Etat sont souvent présentées comme l'un des sujets parmi les plus nobles, les questions afférentes aux rapports des Beaux-Arts et de l'Etat sont surtout «délicates» au sens où les aborder implique d'infinis ménagements. L'on n'en finirait pas de citer les précautions oratoires des parlementaires engageant leur intervention à la chambre par un préambule qui en souligne par avance la modestie et fait valoir en une figure rhétorique obligée l'«ignorance» dans laquelle ils se trouvent de ces choses «complexes» que sont les dossiers des Beaux-Arts. Ainsi, malgré le lyrisme des déclarations de principe, les atermoiements et la modestie affichée l'emportent-elles sur les jugements péremptoires et les propos définitifs. Cette position en retrait, produit de l'intériorisation d'une illégitimité systématiquement construite par les agents du champ artistique, contribue à la produire en retour. On le voit à partir des modes de structuration des discours politiques comme dans les euphémisations qu'ils organisent.

La prudence qui régit l'intervention des agents du champ politico-administratif dans le domaine artistique est en premier lieu indiquée par la structure de leurs discours. Si l'on considère les documents auxquels ils donnent lieu comme des «formes sociales objectivées» dont la structure ordonne les représentations de l'espace social et, partant, l'organise ${ }^{\mathrm{xxiv}}$, alors l'économie des ouvrages sur l'art et l'Etat au sein desquels les logiques des découpages des institutions artistiques l'emportent sur le cadre normatif de l'intervention publique montre bien à quel point les discours politiques eux-mêmes reproduisent l'effacement des logiques étatiques. 
Ces ouvrages, malgré leurs différences d'origine et de vocation, ont une économie en grande partie commune. Le plan type s'organise à la manière d'un catalogue où se succèdent les différents secteurs artistiques ${ }^{\mathrm{xxv}}$. Ce catalogue n'est pas construit à partir de critères esthétiques, ni tout à fait selon les disciplines artistiques, mais opère une classification selon les institutions sociales concernées. C'est ainsi que les écoles des Beaux-Arts sont distinguées des musées, ou que les théâtres populaires occupent une place à part. A ce catalogue s'ajoutent, dans l'introduction ou la conclusion, et plus encore au fil du texte, des considérations générales sur les buts, l'organisation et les problèmes de l'intervention artistique publique. La répartition par «fonctions» est exceptionnelle : seul l'ancien ministre des Arts Antonin Proust organise son propos autour des trois pôles que sont l'enseignement, la conservation et enfin la construction et la décoration des bâtiments ${ }^{\mathrm{xxvi}}$. Autant dire que le traitement de la question de l'art et de l'Etat est fort peu structuré par des logiques propres à l'intervention publique.

En second lieu, la prudence qui résulte de l'intériorisation des contraintes qui pèsent sur l'intervention artistique publique est ostensiblement et systématiquement marquée dans les discours. Cette prudence consiste, tout d'abord, à indiquer, pour les neutraliser ou s'en prémunir, les oppositions à l'intervention publique. De manière prétendument neutre, pour faire valoir l'actualité et le piquant du sujet; à la façon de prolepses, pour déjouer par avance les objections ; sur un mode dénonciateur, pour délégitimer les accusateurs et ainsi légitimer les accusés : il n'est pas de propos sur les Beaux-Arts et l'Etat qui ne mentionne le caractère polémique du sujet. Ainsi, pour prendre des exemples d'auteurs et de textes aux statuts différents, l'ancien ministre des Arts Antonin Proust évoque ironiquement la proposition répétée chaque année de supprimer la subvention pour les concerts et les théâtres $^{x x v i i}$, et Nicolas Boussu, dans ce qui est présenté comme «une monographie des services publics», commence son étude par la mention des attaques de toutes parts dont fait l'objet l'administration des Beaux-Arts.

«Peu d'administrations ont été plus décriées que l'administration des Beaux-Arts»écrit-il, et ces critiques proviennent des endroits les plus variés de l'espace des prises de position politiques et sociales. Cette administration doit en effet faire face aux attaques du parti qui vise à «la destruction de toutes les forces vives du pays» et qui «demande la suppression de la police des mœurs», mais aussi à celles des adversaires de l'interventionnisme et à celles de ceux qui considèrent l'art comme un luxe $\mathrm{x}^{\mathrm{xxvii}}$. 
Ensuite, les producteurs de discours sur l'intervention publique n'ont de cesse d'affirmer le caractère nécessairement modéré de leurs théorisations. Cette modération est tout entière contenue dans la formule du juriste Pierre Bossuet, et que font leur l'ensemble des auteurs : entre Etat-gendarme et Etat-providence, entre liberté totale et autorité contraignante, in medio veritas ${ }^{\text {xxix }}$. Ainsi, la présentation des différentes «écoles» de pensée s'intègre toujours dans une démonstration qui tend à faire opter pour une voie médiane, pragmatique et «modeste». L'exemple le plus clair en est donné par Couyba ${ }^{\mathrm{xxx}}$, qui formule de manière systématique les principes d'oppositions généralement reconnus. Sinon sa conclusion, au moins sa manière de voir est à l'époque largement partagée, comme en témoignent les présentations identiques que l'on peut trouver tant dans la presse que dans les prises de positions d'agents du champ politique fort éloignés les uns des autres ${ }^{\mathrm{xxxi}}$.

Cette prudence doctrinale, qui fait toujours préférer les solutions «modestes» aux options théoriques «excessives», trouve son prolongement dans les appels à la prudence qui prédominent dans les prescriptions des attitudes à adopter en la matière. En plus des contraintes liées à la nécessaire prise en compte des règles du champ artistique, s'ajoute ici la contrainte proprement politique qui oblige ceux qui prétendent incarner la «République» et la «Démocratie» à se démarquer des pratiques des régimes antérieurs. Ainsi, Antonin Proust fait valoir que l'intervention ne doit pas être la «direction» des arts, mais «une collaboration étroite, une sorte de pacte de confiance entre l'action publique et l'action privée» ${ }^{\text {xxii }}$. De même, Couyba souhaite que, dans le prolongement de ses considérations «théoriques», la pratique de l'intervention artistique soit respectueuse tant du principe de l'autorité que de celui de la liberté : «C'est, ce semble, à la conciliation de ces deux principes que doit s'efforcer, dans l'état actuel de la civilisation, tout individu et toute collectivité. C'est en tout cas la tâche que, selon nous, doivent, en attendant mieux, se proposer tous ceux qui sont chargés d'étudier et d'administrer les rapports de l'art et de $l^{\prime} E$ Etat.. ${ }^{\text {xxxiii }}$

\section{L'éclatement des formes de justification}


L'illégitimité de l'intervention artistique publique, intériorisée par les agents placés en position de la produire, ne les oblige pas seulement à la prudence : elle affecte le statut de leurs discours. Plus que des principes normatifs guidant l'action publique, ceux-ci consistent en des réponses à des attaques antérieurement formulées. On comprend dès lors que, plus que des principes normatifs univoques, les discours politiques sur l'intervention publique pour les arts puissent constituer un ensemble à la fois composite et labile de justifications: leur logique de production ne se laisse guère envisager comme la formulation «d'idées» préexistantes, ni même comme le résultat d'une mobilisation autour d'une quelconque «cause», mais procède davantage d'une position défensive à l'égard d'attaques multiformes et changeantes, appelant par conséquent des réponses elles-mêmes multiformes et changeantes.

C'est contre l'argument selon lequel les Beaux-Arts constituent l'apanage exclusif de la sphère et de l'initiative privées que les défenseurs de l'intervention publique tendent à démontrer la nécessité du «devoir d'assistance» que se doit d'assurer l'Etat dès lors que l'évergétisme et le mécénat des «classes riches» sont reconnus comme insuffisants pour assurer la protection des artistes. Conformément aux systèmes qui se mettent progressivement en place à partir de la fin du XIXe siècle, il faut donc porter «assistance» aux artistes et leur assurer la protection sociale par des financements publics. Ainsi, le directeur des Beaux-Arts Gustave Larroumet s'adressant aux élèves de l'Ecole des BeauxArts peut-il être assuré de recueillir l'assentiment général lorsqu'il justifie «la tutelle de l'Etat en matière d'art» par la nécessité de son assistance auprès des artistes.

«L'Etat, dit-il, doit réparer «ces cruelles injustices nées de l'aveugle fatalité des choses et de l'insuffisante éducation artistique du public, qui conduisent à la pauvreté et à la souffrance un trop grand nombre d'entre vous, et des plus méritants. Si la tutelle de l'Etat, en matière d'art, avait besoin d'être justifiée, ne serait-ce pas là un argument décisif ? ${ }^{\mathrm{xxxiv}}{ }_{\text {». }}$

Contre l'argument de l'«inutilité» des Beaux-Arts, il faut, pour faire valoir le bien-fondé de l'intervention publique, démontrer tout l'intérêt qui s'y attache. D'une part, ils forment un secteur économique producteur de richesses, d'autre part, ils induisent des effets qui profitent à nombre d'autres activités économiques, et donc à la collectivité dans son ensemble. Le schème économique ne se limite donc pas à l'assistance charitable; il se réfère également à l'intérêt bien compris des agents économiques, et plus généralement à 
celui de la collectivité. D'Antonin Proust à Gustave Larroumet, des rapporteurs du budget aux théoriciens de l'administration des Beaux-Arts, le schème du complément au système économique fait ainsi partie du point de passage obligé des justifications de l'intervention.

Cette forme de justification est particulièrement présente lors de la création du ministère Proust qui, en réalisant «l'unité des arts» établit un lien étroit entre «les arts et l'industrie» et peut donc prétendre servir les intérêts économiques du pays. Le rapport de Gambetta au président de la République pour la création du ministère des Arts est largement construit autour de cette argument : «l'assistance que l'art peut donner à l'industrie a une importance considérable au point de vue économique et social, puisqu'elle transforme les conditions de travail et exerce une influence décisive sur les forces productives d'une nation.» ${ }^{\mathrm{xxv}}$

Les efforts déployés pour faire valoir l'«utilité» des Beaux-Arts ne se cantonnent pas à des arguments strictement économiques; l'«utilité indirecte ${ }^{\mathrm{xxxvi}} \gg$ dont parlent Gambetta et d'autres à sa suite est censée exercer ses effets positifs sur la «grandeur» et sur l'«état moral» de la nation, et partant doit être favorisée par l'Etat. La «grandeur des nations» se mesure à «l'effort artistique», fait valoir Couyba en reprenant un argument classique, qui permet de mettre entre parenthèses les luttes multiples dont les Beaux-Arts sont l'enjeu ${ }^{\text {xxxvii }}$. Quant à l'argument de la moralisation des masses, il compte parmi les plus fréquemment utilisés. Ainsi, lorsque les juristes Gustave Dupré et Paul Ollendorff cherchent à réfuter la «doctrine libérale» hostile à l'intervention publique ${ }^{\mathrm{xxxviii}}$, ils lui opposent des principes issus de la tradition juridique de la préservation de la morale publique : «laisser faire, c'est laisser passer la décadence ${ }^{\mathrm{xxxix}} \gg$. C’est que «l'Etat, a besoin de l'art pour travailler avec lui à l'éducation du goût et de l'esprit publics, pour faire pénétrer dans le sein des masses, avec le sentiment du beau, un esprit de paix, d'ordre et de progrès ${ }^{\mathrm{xl}} \gg$. C'est en particulier le cas pour les spectacles, réputés exercer une forte «influence sociale», trop souvent «négative»-comme c'est le cas dans les Cafés-Concerts. Il faut dès lors, pour préserver l'ordre et la morale publique; lutter contre cette influence perverse et utiliser à l'inverse le «rôle social du théâtre» afin de propager les bonnes mœurs ${ }^{x l i}$.

«Le but social de l'art scénique, celui qui semble surtout justifier le rôle que nous avons revendiqué pour l'Etat, c'est l'éducation morale et intellectuelle de la foule. Or, il est évident que c'est surtout pour la population ouvrière, à demi illettrée que ce rôle sera utile. [...] L'Etat, tuteur naturel des faibles, doit protéger ces derniers contre ce danger de démoralisation [les cafés concerts]. La censure est impuissante. [...] Le seul moyen de réagir contre ce danger, c'est d'offrir à l'ouvrier un spectacle plus intéressant, à sa portée et à meilleur marché de nature à former son goût et à le détourner des divertissements grossiers ${ }^{\mathrm{xlii}} \gg$. 
Les paradoxes de l'argument démocratique

C'est contre l'accusation récurrente du caractère inégalitaire de l'accès aux Beaux-Arts que se forge peu à peu la référence centrale de l'intervention publique en la matière : l'objectif de «démocratisation» xliii. Produit par et pour la controverse politique, l'argument démocratique ne revêt pas l'unité et la cohérence originelles d'une référence construite par un groupe fortement mobilisé, avec le travail d'exclusion et de précision que cela suppose. Il retire au contraire de ces conditions de production un caractère pour le moins hybride, utilisé par des agents aux positions fortement diversifiées, et à partir de formes antérieurement produites elles-mêmes très diverses (rhétorique de la moralisation des masses, arguments hygiénistes de la lutte contre les fléaux sociaux, visées éducatives ou impératif de l'égalité fiscale, etc.) Il est en cela plus généralement exemplaire des discours politiques sur l'art au tournant du siècle qui, produits essentiellement dans la logique des attaques subies par l'intervention publique pour les Beaux-Arts, contribuent par leur caractère hybride et multiforme à entretenir l'incertitude qui est à leur principe.

Le nombre des publications mêlant les termes d'«art» et de «démocratie» comme les rapports budgétaires affirmant rituellement la nécessité d'«étendre les bienfaits de la démocratie à l'art ${ }^{\text {xliv }}$ » suffisent à le montrer : l'association de ces deux vocables est une figure habituelle du langage politique du tournant du siècle. Habituelle, elle l'est au point de pouvoir être raillée comme un lieu commun des discours pour banquets : «l'art et la démocratie, tel était toujours le thème aux variations infinies» ironise Paul Léon à propos des allocutions routinières de secrétaire d'Etat aux Beaux-Arts Dujardin-Beaumetz ${ }^{\mathrm{xlv}}$. Habituel voire routinier, l'argument n'est pas l'apanage d'une catégorie particulière d'agents du champ politique. Plus que des déterminations sociales ou idéologiques, c'est l'obligation dans laquelle se trouvent les agents de devoir produire une justification à l'intervention publique qui explique la mobilisation du schème de la démocratisation. Les contraintes liées à la position se font ainsi indirectement sentir, par l'intermédiaire des situations de justification qu'elles produisent : la position occupée peut, de manière variable selon les 
configurations, entraîner l'agent qui l'occupe à devoir justifier l'intervention publique pour les arts, et c'est cette situation qui produit le recours à l'argument démocratique. Le rapport qu'entretient la prise de position avec la position occupée est donc ici indirect et variable, alors que celui qui la lie à la situation se dessine beaucoup plus nettement. C'est ainsi que la mobilisation de l'argument prosélyte est au tournant du siècle plus le fait de conservateurs et de représentants des catégories socialement élevées -qui, parce que les logiques de la délégation politique les conduisent à soutenir le financement public des institutions artistiques sont contraints d'en produire la justification- que des agents prétendant représenter les intérêts des classes populaires et idéologiquement proches des références démopédiques ${ }^{\text {xlvi }}$.

La référence à la «démocratisation» constitue le retournement des attaques auxquelles elle répond. La sempiternelle critique de l'inégalité dans la jouissance des choses de l'art qui fonde l'une des principales attaques contre l'intervention publique est en effet convertie en argument qui établit l'objectif même de cette intervention : réduire les inégalités. Les modalités d'accomplissement de ce retournement sont particulièrement visibles dans les controverses qui, à propos de financement public des Beaux-Arts, prennent la question de l'égalité des contribuables pour objet. Les contradicteurs se retrouvent pour affirmer que l'égalité devant l'impôt doit avoir pour corollaire l'égalité des citoyens dans l'accès aux services financés par l'argent public. Mais alors que - les comptes-rendus des séances parlementaires ou municipales en témoignent - de nombreux élus dénoncent l'injustice qu'il y a à «faire payer à tous le plaisir de quelques uns», les défenseurs de l'intervention publique reprennent l'argument, pour, plutôt que d'en déduire la suppression du financement public, l'assortir d'une obligation supplémentaire : celle qui consiste à remplir les conditions nécessaires à ce que ce financement bénéficie effectivement à tous.

Couyba, tout particulièrement, défend cette position. Au cours de la séance à la Chambre des députés du 5 mars 1902, il s'accorde à dire qu'«il n'est pas juste que le pauvre, déjà si écrasé par toutes les iniquités fiscales et sociales, continue à payer pour la plus grande joie du riche». Mais, plutôt, comme le font certains, que de réfuter l'argument au nom du devoir de «solidarité nationale $\left.{ }^{\text {xlvii }}\right\rangle($ sic), il fait de «l'injustice» la raison d'une action nouvelle, et partant la justification de l'intervention. Ainsi, dans un entretien au Petit Parisien, après avoir rappelé qu'il n'est pas normal que les pauvres paient pour les plaisirs des riches, il affirme la nécessité de créer des théâtres pour les ouvriers et en fait une obligation pour les pouvoirs publics ${ }^{\text {xlviii }}$. On retrouve ce même argument, sous une forme métaphorique fréquente : celle de la chère. «Au banquet de l'art, tous les citoyens doivent être admis puisqu'ils en font les frais. Or, étant donné les prix actuels des 
places des théâtres nationaux, ces agapes artistiques ne laissent même pas aux pauvres le droit de ramasser les miettes des repas ${ }^{\text {xlix }} \gg$.

\section{L'illégitimité institutionnalisée, ou la réalisation d'une prophétie}

Construite dans les concurrences entre les champs artistique et politique, intériorisée au point d'entraîner des pratiques qui contribuent à la réaliser, l'illégitimité de l'intervention publique pour les Beaux-Arts marque jusqu'aux institutions d'Etat. Considérées à une date précise, celles-ci accusent l'éclatement qui leur est généralement attribué, non seulement par les jugements postérieurs, mais par ceux-là mêmes qui les font vivre et les pratiquent. Envisagées au cours du temps, elles apparaissent soumises à d'incessantes fluctuations beaucoup plus qu'à un processus d'institutionnalisation qui leur conférerait ce «naturel» des conventions d'Etat progressivement construit dans la cristallisation des pratiques, des références et des classements.

Cet éclatement et ces fluctuations ne sont pas sans rapports avec les processus de délégitimation de l'intervention publique précédemment décrits. Ils peuvent apparaître comme leurs effets : sans cesse dénoncée, la «politique» des Beaux-Arts ne suscite pas les investissements - à tous les sens du terme, y compris d'《investissements de forme ${ }^{1} »-$ de la part du personnel gouvernemental et administratif qui seuls permettraient de lui conférer une place au sein de la panoplie des «fonctions» d'Etat. Mais la réciproque est également vraie: l'incertitude des regroupements opérés dans les nomenclatures administratives, l'instabilité des positions des fonctionnaires chargés des Beaux-Arts ou encore l'absence de département ministériel autonome comptent parmi les éléments mobilisés pour dénoncer l'inanité de l'intervention publique.

\section{Constructions institutionnelles et déficit de légitimité}

L'incertitude des constructions institutionnelles des Beaux-Arts, pour partie produite par le déficit de légitimité de l'intervention publique dans ce domaine, conduit à le reproduire en retour. Les classifications équivoques opérées par et pour l'intervention publique 
témoignent des contraintes qui pèsent sur sa formalisation en même temps qu'elles entravent pratiquement sa conduite.La forme particulière de classification que constituent les nomenclatures budgétaires produit moins l'articulation cohérente d'un ensemble d'éléments formant une «politique» qui les englobe qu'une liste d'objets hétéroclites à la configuration mouvante et dont la logique de constitution paraît incertaine, aux dires mêmes de ceux qui ont à en connaître. Le budget des Beaux-Arts, qui forme la deuxième section du budget de l'Instruction publique, se présente sous une forme relativement distincte des autres chapitres du budget de l'Etat. Il comprend un nombre important et croissant d'articles : 64 en 1882, 68 en 1907, 104 en 1913 ${ }^{\text {li }}$. Moins constituée sur la base d'une taxinomie préétablie que par les effets croisés de la sédimentation des institutions du passé et de l'addition de chapitres divers en quête d'affectation, la structure du budget des Beaux-Arts pose problème à qui voudrait connaître la «politique artistique» de l'Etat.

Le contrôle budgétaire exercé par les députés leur donne l'occasion d'éprouver et de dénoncer -ici sur un mode ironique- les difficultés quant aux opérations de classement dont font l'objet les dossiers des BeauxArts. Le député et artiste Jules Buisson relate ainsi en séance ses problèmes à la lecture des documents budgétaires. "Quand j'ai voulu, par exemple, rechercher sur quel chapitre étaient payés les inspecteurs des Beaux-Arts, je suis allé tout naturellement au personnel de l'administration centrale, puis au personnel des musées. Or, dans ces chapitres, nulle trace du traitement attribué à ces fonctionnaires. C'est au chapitre 40, et sous la rubrique "Ouvrages d'art et décorations des édifices publics", qu'il a fallu que nous allions les retrouver. L'Assemblée imaginerait difficilement tout ce qui est compris dans ce chapitre 40, tout ce qui est payé dans ce chapitre 40. Je lui épargne les détails : je n'en emprunte qu'un à un autre chapitre, le chapitre 46. Qui de vous, messieurs, eût deviné que, sous ce titre Indemnités des missions, se cachent les modestes frais de factage et de transport payés pour le voyage des objets d'art dans les palais et résidences dont la décoration est à la charge de la direction des Beaux-Arts ? ${ }^{\text {lii }}$ ». Les mêmes difficultés sont rencontrées par Paul Bert lorsqu'il souhaite proposer un amendement au budget. La question est pourtant précise, et devrait a priori trouver sans peine à s'insérer dans un chapitre bien identifié : il s'agit d'ouvrir un crédit pour le déménagement des écuries et magasins de fourrage établis sous la galerie de peinture du Louvre, situation qui, d'après le député, s'avère fortement préjudiciable à la conservation des œuvres. Pourtant, l'imbrication des compétences - les écuries sont militaires, le bâtiment sous l'autorité des Travaux publics, et les tableaux du ressort des Beaux-Arts - reproduite par le budget, rend complexe et incertaine cette opération en apparence simple. Après hésitation quant au choix «du meilleur des ministères», l'amendement se trouve finalement proposé au budget des Beaux-Arts. «Je dois m'excuser un peu de l'avoir rattaché au département des Beaux-Arts. Peut-être dans l'application, quelques difficultés peuvent s'élever; mais j'avoue que j'ai senti [de] l'embarras à savoir où le placer [...]. Mon amendement s'est [trouvé] à la recherche du meilleur des ministères. J'ai cru, dès lors, pouvoir le rattacher directement au ministère des Beaux-Arts ${ }^{\text {liii }} \gg$.

Seconde forme durcie de l'objectivation d'une «politique», les organigrammes de l'administration centrale ne donnent pas plus à voir une intervention stable, homogène et 
unifiée. «Aucun de nos services publics n'a subi plus de modifications que le service des Beaux-Arts, soit dans ses attributions, soit dans ses rapports avec les autres services», affirme Gambetta lorsqu'il présente le projet de création d'un ministère des Arts en 1881. Il suit en cela les constats des rapports des sénateurs Charton dès 1875, puis Lambert de Sainte-Croix en 1878, et en précède beaucoup d'autres, comme celui de DujardinBeaumetz dans son rapport pour le budget 1900. L'administration des Beaux-Arts, qui prend la forme d'une Direction, parfois d'une Direction générale ou d'un Secrétariat général, s'apparente à la juxtaposition de services relativement distincts les uns des autres. La Direction qui remplace, tout de suite après la chute du second Empire, un éphémère ministère des Beaux-Arts, comprend un nombre très variable de bureaux et de services dont les attributions respectives ne sont pas plus stables que précises. «Les titres sont défectueux quand les attributions sont complexes, et c'est ici le cas», notent Dupré et Ollendorf ${ }^{\text {liv }}$. Les bureaux et services des travaux d'art, des musées nationaux, de l'enseignement, des monuments historiques, des bâtiments civils, des conservatoires et des théâtres nationaux, ne connaissent qu'une stabilité précaire dans leurs attributions, changeant régulièrement d'administration de tutelle, et ne bénéficiant que très rarement de l'exclusivité et d'un domaine de compétences qui leur serait entièrement attribué.

\section{Les effets inattendus des instances consultatives}

La multiplication des commissions vient encore compliquer l'ordonnancement du service des Beaux-Arts. La constitution d'instances composées de personnalités «qualifiées» extérieures à l'administration - artistes, critiques, représentants des académies, élus - est ici plus qu'un gage de conformité aux «principes démocratiques»: il marque l'indispensable recours à des agents de légitimation externes. Pour autant, ce recours - massif -, loin de conférer à l'administration des Beaux-Arts l'autorité qui lui fait défaut, contribue à entretenir sa situation précaire. Les divers conseils et commissions, déjà présents sous le second empire, se multiplient à partir du début des années 1870. Une dizaine sont en activité en 1885, près de trente dans l'entre-deux-guerres. Mais ces instances ne sont guère 
mieux considérées dans le champ artistique que ne l'est l'administration à laquelle elles sont censées apporter un crédit : elles reçoivent à l'inverse les accusations récurrentes et contradictoires d'organiser la «tyrannie administrative», de servir les intérêts partisans ou les caprices privés des parlementaires et d'être soumises à la domination des académies et de leurs critères de goût, imposés par le double jeu des artistes académiques introduits dans les commissions et des prétendants (fonctionnaires des Beaux-Arts notamment) qui aspirent à la reconnaissance de l'Institut ${ }^{\mathrm{lv}}$. De plus, la dénonciation de la mainmise des conseils sur l'activité artistique n'apparaît guère fondée, tant ces derniers ne pèsent que de peu de poids sur le traitement public des Beaux-Arts et, partant, sur le fonctionnement du champ artistique. Au total, la multiplication des instances consultatives a affaibli les institutions existantes (les jurys d'artistes, notamment) sans pour autant permettre l'établissement d'un système stable de légitimation ; plus, elle a contribué à déposséder les agents de l'administration de nombre des prérogatives dont ils pourraient se prévaloir et ainsi de la possibilité d'établir la légitimité d'une administration publique de l'art ${ }^{\mathrm{lvi}}$.

La principale parmi ces instances consultatives, le Conseil Supérieur des Beaux-Arts (CSBA) créé en 1875, en fournit une bonne illustration ${ }^{\text {lvii }}$. Si le Conseil a pu jouer un rôle important au début de son existence, il voit son activité décliner rapidement. La périodicité de ses réunions en témoigne : mensuelle à sa création en 1875, elle devient trimestrielle en 1880. A partir de 1887, le Conseil ne siège plus qu'à la demande du ministre qui fixe l'ordre du jour; il n'a donc plus de périodicité régulière ni de compétences établies. Son rôle diminue fortement, et ce au profit de la dilution des processus de décision et non de l'unification d'une instance concurrente. En effet, les commissions se multiplient et parcellisent le travail. Elles s'autonomisent du CSBA : souvent créées en son sein, elles s'en détachent progressivement et deviennent indépendantes ; composées au départ de membres du Conseil, le recrutement devient propre à chacune d'entre elles. Le Conseil se trouve ainsi dépossédé d'une part importante de ses prérogatives. De la sorte, dans la seconde moitié des années 1890, l'essentiel de sa tâche ne consiste plus qu'à examiner les titres des candidats aux bourses de voyage; il ne se réunit plus qu'une fois par an. La diminution du rôle du Conseil est également marquée par les transformations de sa composition, qui subit un 
éclatement croissant: ses membres proviennent de plus en plus d'instances politiques et administratives diverses, sans que ce changement ne s'accompagne du renforcement de l'administration centrale des Beaux-Arts ni d'une spécialisation et de la sanction d'une quelconque compétence spécifique des parlementaires ou fonctionnaires désignés. Le CSBA échoue à s'instaurer comme instance légitime de représentation des artistes au sein du champ politico-administratif: les diverses tendances artistiques sont loin d'être équitablement représentées - le nombre des artistes académiques reste à un niveau important et ne commence à décliner qu'à partir de 1913 - et l'éclatement de son recrutement est bien loin d'en faire un organe spécialisé «représentatif» du champ $\operatorname{artistique}^{\text {lviii }}$.

\section{Les positions précaires des agents de l'administration des Beaux-Arts}

Sans cesse réorganisée et fragilisée plus que stabilisée par la multiplication des instances consultatives, l'administration centrale des Beaux-Arts ne compte que des effectifs relativement réduits. A la fin des années 1870, elle se compose du directeur, de quelques inspecteurs, de cinq ou six chefs et autant de sous-chefs de bureau, et d'une dizaine de commis $^{\text {lix }}$. Le personnel d'encadrement fluctue en fonction des modifications de l'organigramme, et tourne autour d'une dizaine de fonctionnaires jusqu'à la fin du siècle. Ce n'est qu'à partir des années 1910 que les effectifs augmentent sensiblement ${ }^{\text {lx }}$. Cette augmentation des effectifs accompagne la codification tardive d'un règlement du personnel, fixant les modalités de recrutement, les mises en congé et les conditions d'avancement ${ }^{1 x i}$. L'instabilité des positions des agents de l'administration des Beaux-Arts est rendue particulièrement visible par les difficultés que rencontre son directeur ${ }^{1 x i i}$. Créé en même temps que la Direction en 1870, le poste de Directeur est supprimé en 1905, puis rétabli en 1919. Dix directeurs se succèdent de 1870 à 1905 , certains ne restant en poste que quelques mois. La diversité des profils et des trajectoires tout comme les témoignages qu'ils laissent dans leurs écrits indiquent la difficulté à tenir une position aussi décriée par les agents du champ artistique que mal établie dans le champ administratif. 
L'échec de la constitution d'un corps d'inspection spécifique à l'administration des BeauxArts indique bien que celle-ci ne connaît pas une institutionnalisation comparable à celle qui intervient à la même époque dans d'autres domaines. Le premier poste d'inspecteur est créé sous la monarchie de juillet; les effectifs fluctuent pour atteindre le nombre de sept inspecteurs et deux inspecteurs généraux à la fin du second empire ; mais la réorganisation républicaine de l'administration des Beaux-Arts en 1872 tend à supprimer ces postes et les réduit à quatre inspecteurs. Recrutant parmi les institutions de l'élite intellectuelle (Collège de France, Institut), les critiques et historiens d'art reconnus, ou les hauts fonctionnaires, l'inspection des Beaux-Arts constitue une charge annexe assumée par des agents aux positions élevées, et qui ne perçoivent pour cela au mieux qu'une faible indemnité. Elle est bien loin, autrement dit, de constituer un corps susceptible d'unifier une administration, à l'instar des finances ou des affaires étrangères. De plus, si à la fin des années 1870, plusieurs mesures paraissent s'acheminer vers la formation d'un corps (la création de dixsept postes d'inspecteurs est prévue par la réforme de l'administration des Beaux-Arts en 1878, un corps d'inspecteurs de l'enseignement du dessin est créé l'année suivante), chaque débat budgétaire à ce sujet tend à réduire les effectifs, et à faire assumer le travail d'inspection par le personnel administratif des Beaux-Arts. Le débat fait long feu, entre les partisans - qu'on trouve pour l'essentiel parmi les administrativistes et les fonctionnaires d'une inspection générale, seule capable à leurs yeux d'établir la légitimité de l'administration des Beaux-Arts, et ses adversaires -des parlementaires pour la pluparthostiles à la création d'un corps selon eux inefficace et qui surtout entraînerait des dépenses supplémentaires au budget. Il conduit à la suppression de l'inspection des théâtres en 1905, et, par voie d'extinction, de l'inspection des Beaux-Arts deux ans plus tard. En 1913, l'inspection se cantonne alors pour l'essentiel à l'enseignement (six inspecteurs de l'enseignement musical, treize inspecteurs de l'enseignement du dessin), l'inspection des Beaux-Arts se trouvant réduite à l'inspecteur général et un inspecteur.

\section{Un ministère introuvable}


Aux difficultés inhérentes à la production administrative des Beaux-Arts, les débats sur le bien-fondé de la création d'un département ministériel autonome ajoutent les obstacles plus étroitement politiques qui tiennent à la suspicion prégnante des usages partisans de l'art et à la dénonciation récurrente de l'incompétence des responsables politiques en la matière. L'existence d'une administration, pourtant souvent placée sous la direction d'un fonctionnaire issu du champ littéraire, soulève déjà des protestations contre le dirigisme borné ; un ministère, et donc la désignation d'un agent politique par définition non spécialiste comme responsable des arts dans le pays suscite inévitablement des protestations plus véhémentes encore: s'agissant d'un ministère, l'épouvantail de la politisation de l'art vient redoubler la difficulté à en faire une catégorie légitime de l'intervention publique.

Il n'existe donc pas - sauf une exception - sous la IIIe république de ministère spécifiquement consacré aux Beaux-Arts. Après la suppression du bref ministère des Beaux-Arts des derniers mois du second empire, un simple sous-secrétariat d'Etat est créé en 1879 et rattaché au ministère de l'Instruction publique ; mais à sa place subalterne dans la hiérarchie gouvernementale s'ajoute le caractère épisodique et souvent éphémère du poste, l'auxiliaire du ministre de l'Instruction publique n'étant fréquemment pas affecté spécifiquement aux Beaux-Arts, comme c'est le cas de 1882 à 1885, et de 1886 à 1905 . Seul Dujardin-Baumetz, sous-secrétaire d'Etat aux Beaux-Arts de 1905 à 1911 connaît une rare longévité. L'exception parmi ces «strapontins» ministériels est celle du ministère des Arts confié à Antonin Proust au sein du cabinet Gambetta. Sa double particularité est de confier le domaine artistique à un ministre de plein exercice et d'opérer un regroupement inédit qui tend à transformer la représentation de l'intervention publique et de son objet : il s'agit des «Arts» dans leur ensemble, et non plus des seuls «Beaux-Arts», ce qui inclut les pans généralement délaissés de l'artisanat, des arts décoratifs et des industries d'art, et imprime à l'intervention publique un tour à la fois plus économique et plus vulgarisateur, puisqu'il s'agit alors de soutenir un secteur d'activité et d'introduire - grâce à l'art dit industriel - le «beau» dans les objets usuels. Mais le bref ministère des Arts ne survit pas au 
«grand ministère» de Gambetta - de novembre 1881 à janvier 1882 - et n’est pas reconstitué par la suite.

La création d'un ministère, seule en mesure de permettre les conditions de l'établissement d'une autorité politique est certes, dans des conjonctures diverses et par des agents aux positions différentes, présentée à plusieurs reprises comme une nécessité. Des appels à une meilleure division de travail gouvernemental sont répétés après la suppression du ministère des Arts ${ }^{\text {lxiii }}$, puis plusieurs années après : il faut, dit Joseph Paul-Boncour, «un vrai ministère, constitué avec tous ses organes, mettant la responsabilité là où est l'autorité, jouissant vis-à-vis des autres et du Parlement lui-même du prestige dont a besoin une défense plus efficace de l'art français ${ }^{\text {lxiv }}{ }$. Toutefois, si la nécessité d'un ministère peut être affirmée des années 1880 aux années 1910, autant d'arguments contraires s'y opposent et peuvent être aussi longtemps répétés. Un ministère chargé des questions artistiques peut en effet être considéré comme «un luxe de spécialisation» ${ }^{\mathrm{lxv}}$, argument de la rationalité administrative derrière lequel se profilent les deux épouvantails maintes fois brandis de l'art par essence élitiste et des risques de sa politisation. La représentation du caractère intrinsèquement hiérarchique des «œuvres de l'esprit», historiquement construite et objectivement réalisée, tout comme le spectre de l'héritage de l'Ancien régime constituent des freins à cette construction institutionnelle. L'opposition à la création d'un ministère s'exprime également sur le registre de l'autonomie du champ artistique, qui se décline en appel au respect de la liberté des artistes, en dénonciation de toute «politisation», et en opposition entre responsabilité politique et compétence esthétique. Ainsi, la dénégation de la légitimité de l'intervention artistique publique entrave-t-elle efficacement l'institutionnalisation d'une «politique»; elle contribue à empêcher la constitution de formes institutionnelles spécifiques et partant à maintenir agents et administrations de l'Etat dans une position précaire qui réalise objectivement la prophétie de leur illégitimité. Et ce déficit de légitimité est d'autant plus fort qu'il est en quelque sorte juridiquement codifié, et donc si l'on peut dire garanti par l'Etat. De fait, non seulement l'intervention publique pour l'art ne donne pas lieu à l'élaboration une théorie du service public, mais une doctrine construite à propos des théâtres lui dénie au contraire cette qualification. Un arrêt 
de la cour de cassation en 1901 refuse une première fois la qualité de service public à l'Opéra-Comique. Une décision du conseil d'Etat en 1916 élabore ensuite une jurisprudence faisant des spectacles une chose par nature contraire à cette notion. Par où l'on retrouve sous une autre forme l'antagonisme entre l'art et l'Etat évoqué au début de cet article...

Contre les simplifications d'une histoire héroïque ou dénonciatrice, on a essayé de montrer que la production d'une politique ne se résout pas dans la «volonté» d'individus agissants mais s'analyse - dans le monde des choses de l'art où le mythe du héros créateur est particulièrement prégnant tout autant qu'ailleurs - dans la complexité des configurations historiques qui rendent la «volonté» pensable et possible. On a en ce sens tenté de restituer la dialectique de l'histoire incorporée dans des dispositions - celles des agents du champ artistique comme celles des agents des champs administratif et politique - et de l'histoire objectivée dans des institutions - administration des Beaux-Arts, ministères, etc. L'on espère ainsi avoir contribué à prendre la mesure des transformations nécessaires à l'émergence et à l'institutionnalisation des choses de l'art et de la culture comme objets spécifiques de l'intervention publique, ce qui est aussi une manière de contribuer à l'analyse de la genèse de l'Etat.

${ }^{\mathrm{i}}$ Cet article reprend des éléments de la première partie de ma thèse, La culture comme catégorie d'intervention publique. Genèses et mises en forme d'une politique, thèse de Doctorat en science politique sous la direction de M. Offerlé, IEP-Lyon II, 1994, 810 p.

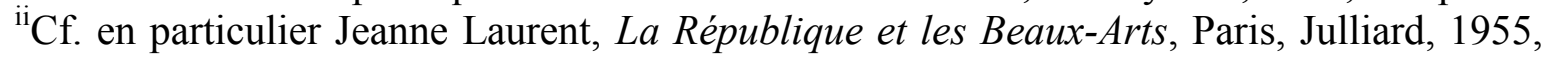
226 p. Nombre de discours du premier ministre des Affaires culturelles, André Malraux, reprennent cet anathème, tout comme les hauts fonctionnaires du ministère ou les membres des commissions culturelles du Plan au début des années 1960.

${ }^{\mathrm{iii}} \mathrm{Cf}$. notamment Pierre Cabanne, Le pouvoir culturel sous la Ve république, Paris, Olivier Orban, 1981, 447 p. ; Michel Durupty, L'Etat et les Beaux-Arts, Bordeaux, Thèse pour le doctorat en droit, Université de Bordeaux, 1964, 697 p. ; Jack Lang, L'Etat et le théâtre, Paris, L.G.D.J., 1968, 373 p.; André-Hubert Mesnard, L'action culturelle des pouvoirs publics, Paris, L.G.D.J., 1969, 544 p.

${ }^{1 v}$ Le premier travail réalisé en ce sens est la thèse soutenue en 1980 et récemment publiée de Pierre Vaisse, La troisième République et les peintres, Paris, Flammarion, 1995, 475 p. L'intérêt de l'ouvrage tient à ce qu'il remet en cause de façon systématique et étayée le récit épique d'une «libération» progressive de l'art au XIXe siècle du «joug» étatique et académique, «légende» propre selon l'auteur à conforter le mythe des héros de l'art moderne et à laisser dans l'ombre des pans entiers de la création artistique. Toutefois, cette 
utile remise en cause vient servir un jugement esthético-politique qui, quoi qu'il cherche à s'en dédouaner, conduit de fait l'auteur à verser dans une forme de réhabilitation.

${ }^{\mathrm{v}}$ Marie-Claude Genet-Delacroix fait ainsi valoir que «le régime a élaboré une stratégie d'investissement artistique et culturel originale et moderne, quoiqu'on en dise. [...] Au vrai la IIIe République a beaucoup innové, plus en tout cas qu'on ne l'a dit.» Marie-Claude Genet-Delacroix, Art et Etat sous la IIIe République. Le système des beaux-arts, 18701940, Paris, Publications de la Sorbonne, 1992, 433 p. (p. 322).

${ }^{\mathrm{vi}} \mathrm{Cf}$. Marc Fumaroli, L'Etat culturel. Essai sur une religion moderne, Paris, Editions de Fallois, 1991, 305 p., notamment p. 70.

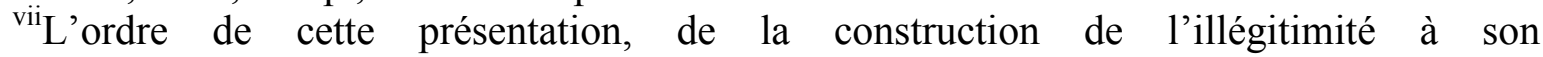
institutionnalisation en passant par son intériorisation, correspond à la logique de démonstration que l'on a tenté d'établir mais ne signifie évidemment pas qu'on le pose comme un schéma de causalité linéaire : ces trois processus s'entretiennent mutuellement plus qu'ils ne se succèdent rigoureusement l'un à l'autre.

viii Pierre Bourdieu, Les règles de l'art. Genèse et structure du champ littéraire, Paris, Seuil, 1992, $481 \mathrm{p}$.

${ }^{\text {ix }}$ On reprend ici la typologie d'Albert Boime, The Academy and French Painting in the Nineteenth Century, New York, Phaidon, 1971, 330 p. Cf. également, à la suite de cet ouvrage, Marie-Claude Genet-Delacroix, «Vies d'artistes : art académique, art officiel et art libre en France à la fin du XIXe siècle», Revue d'Histoire Moderne et Contemporaine, Tome XXXIII, janvier-mars 1986, p. 40-73.

${ }^{\mathrm{x}}$ Réponse à l' 'Enquête sur la séparation des Beaux-Arts et de l'Etat», Les Arts de la Vie, octobre 1904, Première année, Tome II, $n^{\circ} 10$.

${ }^{\mathrm{xi}}$ Ibid. Pour une présentation complète de cette enquête, Cf. notre thèse, La culture comme catégorie d'intervention publique, op. cit., p. 112-122.

${ }^{x i i}$ Sur l'homologie entre les moyens de mobilisation du champ intellectuel et les instances parlementaires et la manière dont ils rentrent en concurrence, Cf. Christophe Charle, Naissance des intellectuels, Paris, Minuit, 1990, 271 p., (p. 116).

${ }^{x i i i}$ Maurice le Blond, commentaire des réponses in «Enquête sur la séparation...», art. cit.

${ }^{\mathrm{xiv}}$ Ibid., p. 216.

${ }^{\mathrm{xv}}$ Ibid., p. 202.

${ }^{\mathrm{xvi}}$ Réponse du Comte Robert de Montesquiou, poète, Ibid., p. 232. Sur la position de Directeur des Beaux-Arts, Cf. notre thèse, op. cit., p. 211-226.

${ }^{x v i i}$ Cf. l'Enquête sur l'Art et l'Etat, art.cit., p. 224.

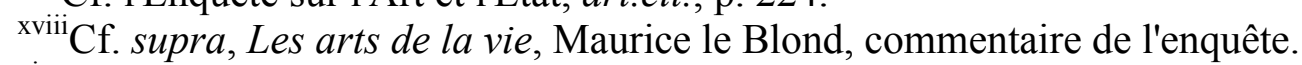

${ }^{\mathrm{xix}}$ Paul Leroi, «Le sous-secrétariat des beaux-arts», L'Art, Tome III, 1881, p. 231-234. (p. 231.)

${ }^{\mathrm{xx}}$ Ibid.

${ }^{x x i}$ Paul Léon, Du Palais-Royal au palais-Bourbon, Paris, Albin Michel, 1947, 302 p., (p. 222). Paul Léon, chef de cabinet du sous-secrétaire d'Etat aux Beaux-Arts DujardinBeaumetz en 1905, reste fonctionnaire aux beaux-arts après le départ de ce dernier en 1911. Il devient chef de service en 1912, puis directeur des Beaux-Arts lors du rétablissement de ce poste en 1919.

${ }^{x x i i} \mathrm{Cf}$. sur ce point, à propos de l'affaire Dreyfus, Christophe Charle, «Champ littéraire et champ du pouvoir. Les écrivains et l'affaire Dreyfus», Annales ESC, nº 2, mars-avril 1977, p. 240-264. Cf. plus généralement Louis Pinto, «La vocation de l'universel. La représentation de l'intellectuel vers 1900», Actes de la recherche en sciences sociales, $\mathrm{n}^{\circ}$ 55, 1984, p. 23-32, et Pierre Bourdieu, Les règles de l'art, op. cit., notamment p. 185 et suivantes. 
${ }^{x x i i i}$ Sont notamment avancés les stigmates de l'héritage du second Empire et plus généralement de l'ancien régime, le poids des intérêts personnels, les inégalités sociales devant l'art.

${ }^{\mathrm{xxiv}}$ Sur ces questions, cf. Robert Salais et al., L'invention du chômage. Histoire et transformations d'une catégorie en France des années 1890 aux années 1980, Paris, PUF, 1986, notamment p. 189. voir aussi Jack Goody, La raison graphique, Paris, Minuit, 1979, qui montre le rôle et les effets sociaux de l'écriture dans les formes d'objectivation sociale, et aussi dans une perspective ethnométhodologique l'article de Dorothy E. Smith, «Les textes comme instruments de l'organisation sociale», Revue Internationale des Sciences Sociales, $\mathrm{n}^{\circ}$ 99, 1984 (1), p. 59-75.

${ }^{\mathrm{xxv}}$ Cf. par exemple L'Art et la démocratie, du député Charles-Maurice Couyba, dont le sous-titre - Les écoles, les théâtres, les manufactures, les musées, les monuments - indique la logique de construction, et l'ouvrage de Joseph Paul-Boncour qui, dix ans plus tard, adopte un titre et un plan à peu près identiques. Charles-Maurice Couyba, L'Art et la démocratie, Paris, Flammarion, 1902, 363 p. ; Joseph Paul-Boncour, Art et démocratie, Paris, Librairie Paul Ollendorff, 1912, 318 p.

${ }^{x x v i}$ Antonin Proust, L'Art sous la République, Paris, Bibliothèque Charpentier, 1892, $276 \mathrm{p}$. Il reprend ainsi comme plan de son ouvrage l'organigramme de son éphémère ministère. $\mathrm{A}$ ce propos, cf. infra.

${ }^{x x v i i}$ Antonin Proust, L'Art sous la République, op. cit.

${ }^{x x v i i i}$ Nicolas Boussu, L'administration des Beaux-Arts, études administratives, Paris, 1877 , éditions E. Boltenweck, 427 p.

${ }^{\mathrm{xxix}}$ Pierre Bossuet, Histoire administrative des rapports des théâtres et de l'Etat, Thèse de doctorat, Imprimerie Henri Jouve, Paris, 1909, 505 p., (p. 12). Cf. aussi la thèse d'économie politique sur «le rôle de l'Etat en matière scénique» de Paul Sorin, qui tente d'abolir les oppositions entre «libéralisme» et «étatisme». Paul Sorin, Du rôle de l'Etat en matière scénique, Thèse de doctorat en Economie Politique, Faculté de Droit, Paris, éditions Arthur Rousseau, 1902, $215 \mathrm{p}$.

${ }^{\mathrm{xxx}}$ Charles-Maurice Couyba, L'Art et la démocratie, op. cit.

${ }^{x x x i}$ Cf. l'Eclair, du 30 novembre 1901, et le rapport du budget des Beaux-Arts pour 1900, de Dujardin-Beaumetz, qui indiquent dans les mêmes termes les trois écoles distinguées par Couyba. Ce dernier est député de la gauche radicale, alors que Dujardin-Beaumetz est nettement marqué à droite.

${ }^{\mathrm{xxxii}}$ Antonin Proust, L'Art sous la République, op. cit., p. 3.

${ }^{x x x i i i}$ Charles-Maurice Couyba, L'Art et la démocratie, op. cit., p. 7.

${ }^{x x x i v}$ Discours prononcé à l'Ecole des Beaux-Arts, le 25 novembre 1888, reproduit en appendice de Gustave Larroumet, L'Art et l'Etat en France, Paris, Hachette, 1895, 370 p. (p. 330). La dernière phrase, affirmation sous forme interro-négative, indique bien la posture justificative adoptée.

${ }^{\mathrm{xxxv}} \mathrm{Ce}$ passage est cité in Antonin Proust, L'art sous la république, op. cit., p. 11. Cf. aussi Louis de Ronchaud, «De l'encouragement des Beaux-Arts par l'Etat», La Nouvelle Revue, vol. XXXIII, mars-avril 1885. Sur le ministère Proust, Cf. infra, et pour plus de détails, notre thèse $\mathrm{p}$. 155-156 et 233-242.

${ }^{\mathrm{xxxvi}}$ Paul Sorin, Du rôle de l'Etat en matière scénique, op. cit.

${ }^{x x x v i i}$ Charles-Maurice Couyba, L'Art et la démocratie, op. cit., p. 3.

${ }^{x x x v i i i}$ Position exprimée notamment par le député et banquier Edouard Aynard. Cf. «Les Beaux-Arts et l'économie politique», in Léon Say et Joseph Chailley (dir.), Nouveau dictionnaire d'économie politique, Paris, Guillani, 1890, ainsi que ses nombreuses interventions à la chambre, par exemple lors de la discussion budgétaire du 28 novembre 1896. 


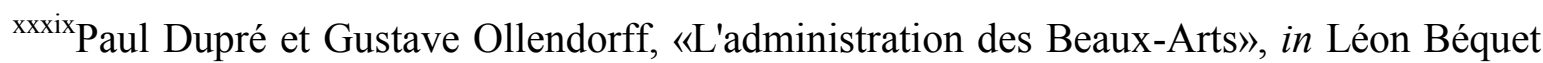
(dir.) Répertoire du Droit Administratif, Paris, éditions Dupont, 1885, p. 70.

${ }^{\mathrm{xl}}$ Louis de Ronchaud, «De l'encouragement...», art. cit., p. 131.

${ }^{x l i}$ L'argument est systématiquement repris par les rapporteurs du budget pour convaincre du bien-fondé des subventions théâtrales. Cf. par exemple le rapport du budget des Beaux-Arts à la chambre pour 1901, par Georges Berger.

${ }^{x l i i}$ Paul Sorin, Du rôle de l'Etat... op. cit., p. 159-161.

${ }^{x l i i i}$ L'on n'aborde ici que l'usage du schème démocratique par les agents du pôle institutionnel du champ politique (parlementaires, responsables gouvernementaux). Pour une restitution des concurrences qui les opposent à cet égard à certaines fractions du champ intellectuel, Cf. notre article «Les prémices de la "démocratisation culturelle". Les intellectuels, l'art et le peuple au tournant du siècle», Politix, n 24, décembre 1993, p. 3656.

${ }^{\text {xliv }}$ Rapport du budget des Beaux-Arts 1912, par Simyan, séance du 12 juillet 1911.

${ }^{\mathrm{xlv}}$ Paul Léon, Du palais-royal... op. cit., p. 91.

${ }^{x l v i}$ Comme le montrent également les débats au conseil municipal de Lyon. Cf. sur ce point notre article «Des "plaisirs bourgeois" aux "droits du peuple", la formation d'un discours politique sur la culture (1884-1930)», Sources, n 26, 1991, p. 43-60.

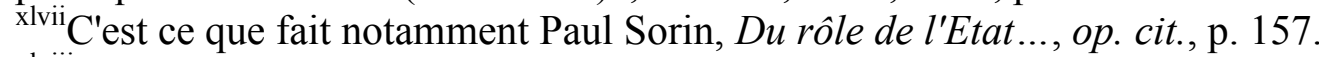

xlviii Le Petit Parisien, 5 décembre 1901.

${ }^{x l i x}$ Charles-Maurice Couyba, L'Art et la démocratie, op. cit., p. 141.

${ }^{1}$ Laurent Thévenot, «Les investissements de forme», in Laurent Thévenot (dir.), Conventions économiques, Paris, CEE-PUF, 1986, p. 21-71.

${ }^{\text {li } A}$ titre de comparaison, le service de l'instruction publique ne compte que 45 chapitres budgétaires en 1882, alors que le domaine couvert est plus vaste et le budget nettement supérieur - les Beaux-Arts représentent alors $16 \%$ du total du ministère de l'Instruction publique.

lii Jules Buisson, séance du 20 mars 1872, J.O. p. 1795, cité in Marie-Claude GenetDelacroix, Art et Etat, op. cit., p. 257-258.

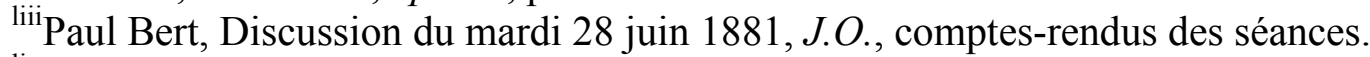

${ }^{\text {liv }}$ Paul Dupré et Gustave Ollendorff, «L'administration des Beaux-Arts», art. cit., p. 47-49.

${ }^{\mathrm{lv}}$ Pour un exemple typique des critiques dont les commissions artistiques font l'objet, Cf. Octave Mirbeau, «Sur les commissions», Le Figaro, 10 août 1890, repris dans le recueil Combats esthétiques, vol. 1, Paris, Séguier, 1993, p. 399-404.

${ }^{1 \mathrm{lvi}} \mathrm{Cf}$. à cet égard les remarques des directeurs des Beaux-Arts Philippe de Chennevières, Rapport adressé à M. le Ministre de l'Instruction publique, des cultes et des Beaux-Arts sur l'administration des arts depuis le 31 décembre 1873 jusqu'au ler janvier 1878, Paris, P. Mouillot, 1878, et Gustave Larroumet, L'art et l'Etat, op. cit., p. 314-315.

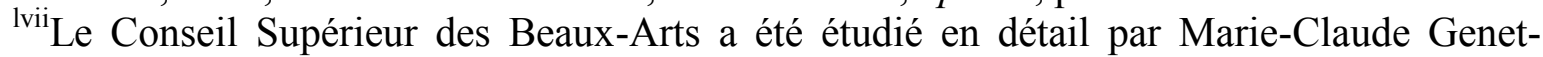
Delacroix dans une perspective tout à fait différente. Cf. notamment Art et Etat, op . cit. ; «Le Conseil Supérieur des Beaux-Arts : histoire et fonction, 1875-1940», Mouvement Social, no 163, 1993.

lviii Témoignent de cet échec les différents projets de réforme inaboutis tendant à concilier au sein du Conseil les principes de la libre expression des artistes et du suffrage universel : le sous-secrétaire d'Etat Dujardin-Beaumetz souhaite instaurer la règle d'un recrutement par voie électorale; le rapporteur du budget de 1911, Joseph Paul-Boncour, propose «la proportionnelle de l'art» pour assurer une représentation équitable. Cf. Marie-Laure Albertini, La politique artistique de Dujardin-Beaumetz devant le parlement et l'opinion, 1905-1912, Mémoire de maîtrise d'histoire, Paris I, 1979, 169 p., et Joseph Paul-Boncour, Art et démocratie, op. cit. 
${ }^{\text {lix }}$ Nicolas Boussu, L'administration des Beaux-Arts..., op. cit.

${ }^{\mathrm{Ix}} \mathrm{Il}$ faut aussi ajouter à ces effectifs le personnel des inspections et des services extérieurs des Beaux-Arts, soit vingt-huit personnes en 1913. Source : Budget général des dépenses de l'exercice 1913, $\mathrm{N}^{\circ} 1846,1912$, Chapitre 4.

${ }^{1 x i} I l$ n'existait pas avant le décret du 1er avril 1909 de texte général, mais seulement une succession de décrets partiels (26 novembre 1897, 6 janvier 1905 et 26 avril 1907).

${ }^{1 x i i} \mathrm{Cf}$. sur ce point notre thèse, p. 211-226.

${ }^{1 x i i i}$ Gustave Larroumet, L'Art et l'Etat en France, op. cit., p. 302-303.

${ }^{1 x i v}$ Joseph Paul-Boncour, Art et démocratie, op. cit., p. 9 et 11.

${ }^{1 \times v}$ M. Racinet, La spécialisation ministérielle, Thèse de Droit, Paris, 1910, cité in Lang Jack, L'Etat et le théâtre, op. cit., p. 53. 